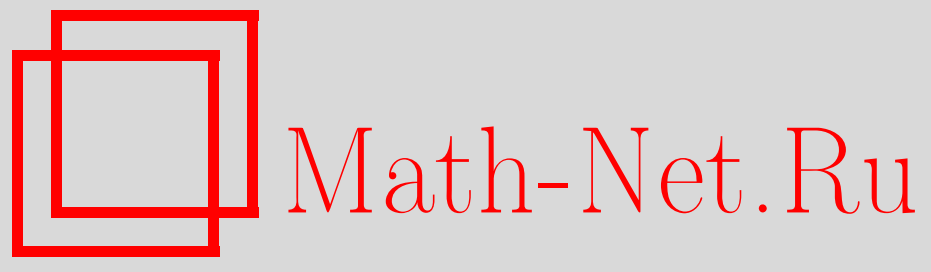

Г. В. Рыжаков, Резольвентные пределы квантовой эволюции открытых систем, Матем. заметки, 2006, том 80, выпуск 3, 476-480

DOI: https://doi.org/10.4213/mzm2837

Использование Общероссийского математического портала Math-Net.Ru подразумевает, что вы прочитали и согласны с пользовательским соглашением http://www . mathnet.ru/rus/agreement

Параметры загрузки:

IP: 54.162 .127 .20

26 апреля 2023 г., 14:47:50

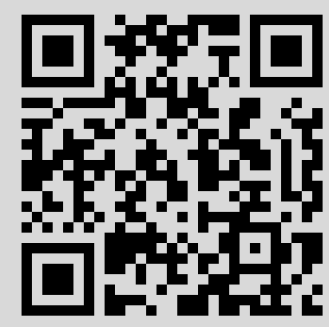




\section{РЕЗОЛЬВЕНТНЫЕ ПРЕДЕЛЫ КВАНТОВОЙ ЭВОЛЮЦИИ ОТКРЫТЫХ СИСТЕМ}

\section{Г. В. Рыжаков}

Пусть $\mathscr{H}$ - сепарабельное гильбертово пространство, $\mathscr{B}(\mathscr{H})$ - алгебра ограниченных операторов, действующих в $\mathscr{H}, \Gamma^{S}\left(L_{2}(\mathbb{R})\right)$ - симметричное фоковское пространство. Рассмотрим уравнение для оператора эволюции $U_{t}$

$$
\frac{d U_{t}}{d t}=i H U_{t}, \quad U(0)=I,
$$

с гамильтонианом $H$, действующего в тензорном произведении $\mathfrak{H}=\mathscr{H} \otimes \Gamma^{S}\left(L_{2}(\mathbb{R})\right)$, где

$$
\begin{aligned}
H & =H_{0} \otimes I-I \otimes i \nabla+H_{\text {int }}, \\
H_{\text {int }} & =K \otimes \Lambda(g)+R \otimes A^{\dagger}(g)+R^{*} \otimes A(g),
\end{aligned}
$$

причем $K^{*}=K, H_{0}^{*}=H_{0}$ и $R$ - ограниченные операторы,

$$
\Lambda(g)=\int d x g(x) a^{\dagger}(x) a(x), \quad A(g)=\int d x \bar{g}(x) a(x), \quad A^{\dagger}(g)=A^{*}(g),
$$

$g \in L_{1}(\mathbb{R}) \cap L_{2}(\mathbb{R}) \cap C(\mathbb{R}), a(x)$ и $a^{\dagger}(x)$ - плотности операторов уничтожения и рождения.

Вместо фиксированной функции $g$, входящей в определение гамильтониана $H_{\mathrm{int}}$, будем рассматривать семейство неотрицательных финитных функций $g_{\alpha}(x) \in C_{0}^{\infty}(\mathbb{R})$, $\alpha \in(0,1]$, которое имеет пределом дельта-функцию:

$$
\lim _{\alpha \rightarrow 0} \int f(x) g_{\alpha}(x) d x=f(0)
$$

для любой непрерывной ограниченной функции $f$, причем диаметр $d_{g}(\alpha)$ носителя функции $g_{\alpha}$ стремится к нулю при $\alpha \rightarrow 0$. С физической точки зрения такой предел соответствует модели точечного взаимодействия системы с окружением. Формальный предел семейства гамильтонианов $\lim _{\alpha \rightarrow 0} H_{\mathrm{int}}^{\alpha}$ является сингулярно возмущенным оператором, так как результат его действия на векторы вида $\psi(v) \otimes r$, где $\psi(v) \in \Gamma^{S}\left(L_{2}(\mathbb{R})\right)$ - когерентный вектор, $v(0)=0, r \in \mathscr{H}$, равен нулю, а линейная оболочка таких векторов плотна в $\mathfrak{H}$.

В дальнейшем, где это не приводит к путанице, будем опускать индекс $\alpha$. Случай, когда операторы $K, R$ и $H_{0}$ коммутируют, рассмотрен в [1]. В настоящей работе рассматривается обобщение на случай некоммутирующих операторов.

Рассмотрим уравнение (1) в представлении взаимодействия, порождаемом оператором $\widehat{H}_{0}=H_{0} \otimes I-I \otimes i \nabla$, не зависящим от $g$ :

$$
\frac{d U(t)}{d t}=i H(t) U(t), \quad U(t)=e^{-i \widehat{H}_{0} t} U_{t} e^{i \widehat{H}_{0} t},
$$

с гамильтонианом

$$
H(t)=e^{-i \widehat{H}_{0} t} H_{\mathrm{int}} e^{i \widehat{H}_{0} t}=K(t) \otimes \Lambda_{t}(g)+R(t) \otimes A_{t}^{\dagger}(g)+R^{*}(t) \otimes A_{t}(g),
$$

где $K(t)$ и $R(t)$ - непрерывные по норме семейства ограниченных операторов

$$
K(t)=e^{-i H_{0} t} K e^{i H_{0} t}, \quad R(t)=e^{-i H_{0} t} R e^{i H_{0} t},
$$

(C) Г.В. РЫжаков, 2006 
а операторы $\Lambda_{t}(g), A_{t}(g)$ и $A_{t}^{\dagger}(g)=A_{t}^{*}(g)$ являются неограниченными:

$$
\begin{aligned}
& \Lambda_{t}(g)=e^{-t \nabla} \Lambda(g) e^{t \nabla}=\int d x g(x-t) a^{\dagger}(x) a(x), \\
& A_{t}(g)=e^{-t \nabla} A(g) e^{t \nabla}=\int d x g(x-t) a(x) .
\end{aligned}
$$

В качестве тотального множества, задающего область определения данных операторов, возьмем линейную оболочку $\mathscr{L}_{\varepsilon}$ множества

$$
E_{\varepsilon}^{S}=\left\{\phi(f): \phi(f) \in E^{S},\|f\|_{L_{2}(\mathbb{R})}<1,|f|<\varepsilon\right\},
$$

где $E^{S}$ - множество когерентных векторов, $0<\varepsilon \leqslant 1$. Линейная оболочка такого множества плотна в $\Gamma^{S}\left(L_{2}(\mathbb{R})\right)[2]$; рассматриваемые операторы - сильно непрерывное по $t$ семейство операторов, действующих из $E_{\varepsilon}^{S}$ в $\Gamma^{S}\left(L_{2}(\mathbb{R})\right)$. С его помощью введем следующую норму в $\mathfrak{H}$ :

$$
p(X)=\sup _{\psi, h}\|X h \otimes \psi\|_{\mathfrak{H}}
$$

где $\psi \in E_{\varepsilon}^{S}, h \in \mathscr{H}$, причем $\|\psi\|_{\Gamma^{S}}=\|h\|_{\mathscr{H}}=1$.

Свойства преднормы для $p(X)$ проверяются непосредственно. Свойство нормы $p(X)=0 \Longrightarrow X=0$ следует из линейности рассматриваемых операторов и тотальности множества $E_{\varepsilon}^{S}$. Норма $p(X)$ определяет локально выпуклую топологию в $\mathfrak{H}$ [3].

Пусть

$$
K_{\Lambda}(t)=\int_{0}^{t} K(s) \Lambda_{s}(g) d s, \quad K_{g}(t, u)=\int_{0}^{t} K(s) g(u-s) d s .
$$

Положим

$$
R(t, u)=e^{-i K_{g}(t, u)} e^{-i K_{\Lambda}(t)} R(t) e^{i K_{\Lambda}(t)}
$$

и рассмотрим семейство операторов

$$
\begin{aligned}
U_{\alpha}(t)=e^{i K_{\Lambda}(t)} & \exp \left\{-\int_{0}^{t} d \tau \int_{0}^{\tau} d s \int d u \widetilde{R}^{*}(\tau, u) \widetilde{R}(s, u) g(u-\tau) g(u-s)\right\} \\
& \times \exp \left\{i \int d w a^{\dagger}(w) \int_{0}^{t} d \tau g(w-\tau) \widetilde{R}(\tau, w)\right\} \\
& \times \exp \left\{i \int d w \int_{0}^{t} d \tau \widetilde{R}^{*}(\tau, w) a(w) g(w-\tau)\right\} .
\end{aligned}
$$

Семейство операторов $U_{\alpha}(t)$ корректно определено на $\mathscr{L}_{\varepsilon}(\mathscr{H})=\mathscr{L}_{\varepsilon} \otimes \mathscr{H}$, его зависимость от $\alpha$ обусловлена семейством $g_{\alpha}$. Найдем асимптотику разрешающего оператора уравнения Шрёдингера с гамильтонианом (4) с точностью до $O\left(t^{2}\right)$.

Теорема 1. Пусть $H(t)$ определено в (4). Тогда для любых единичных векторов $\phi \in E_{\varepsilon}^{S}, \varphi \in \Gamma^{S}\left(L_{2}(\mathbb{R})\right), h_{1}, h_{2} \in \mathscr{H}$ справедлива оченка

$$
\begin{aligned}
\left(h_{2}\right. & \left.\otimes \varphi,\left(U_{\alpha}(t+\Delta t)-\left(1+\int_{t}^{t+\Delta t} H(\tau) d \tau\right) U_{\alpha}(t)\right) h_{1} \otimes \phi\right)_{\mathfrak{H}} \\
& =O\left(\Delta t^{2}\right) C_{1}(\varphi, \phi) C_{3}\left(g_{\alpha}\right)+O(t \Delta t) C_{2}(\varphi, \phi) C_{4}\left(g_{\alpha}\right),
\end{aligned}
$$

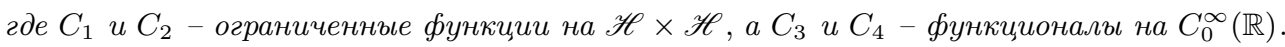

Рассмотрим предел семейства операторов $U_{\alpha}$ при $g_{\alpha} \underset{\alpha \rightarrow 0}{\longrightarrow} \delta(x)$. Выражение для действия предельного оператора на произвольный когерентный вектор $\psi(f)$ можно записать 
явно:

$$
\begin{aligned}
\psi^{t}(f) \stackrel{\text { def }}{=} & \lim _{\alpha \rightarrow 0} U_{\alpha}(t) \psi(f) \\
= & \exp \left\{i\left(H_{0}\right)_{5} t\right\} \exp \left\{-\left(R^{*}(\exp \{i K\}-i K-1)(i K)^{-2} R\right)_{3} t\right\} \\
& \times \exp \left\{i \int_{0}^{t} d \tau\left(R^{*}\left(e^{i K}-1\right)(i K)^{-1}\right)_{1} f(\tau)\right\} \psi\left(f^{t}\right) ;
\end{aligned}
$$

предельная функция $f^{t}(x)$ равна $f^{t}(x)=r(x)(f(x+t)+h(x))$, причем

$$
h(x)=i I_{(-t, 0)}(x)\left(\left(1-e^{-i K}\right)(i K)^{-1} R\right)_{2}, \quad r(x)=\exp \left\{i(K)_{4} I_{(-t, 0)}(x)\right\} .
$$

Индексы у операторов определяют порядок их действия.

Вычислим краевое условие, которому удовлетворяет предельный вектор состояния, а также предельный генератор семейства групп $U_{\alpha}$. Учитывая теорему 1 и тот факт, что генератор (2) не зависит от времени, краевое условие и предельный генератор можно найти, используя семейство $U_{\alpha}(t)$. Определим следующие специальные операторы уничтожения:

$$
A_{ \pm} \psi(v) \stackrel{\text { def }}{=} v( \pm 0) \psi(v), \quad v \in W_{2}^{1}(\mathbb{R} \backslash\{0\}) .
$$

Из (7) следует, что

$$
A_{-} \psi^{t}(f)=\left[\left(e^{i K}\right)_{4} f(t)+i\left(e^{i K}\right)_{4}\left(\left(1-e^{-i K}\right)(i K)^{-1} R\right)_{2}\right] \psi^{t}(f)
$$

и $A_{+} \psi^{t}(f)=f(t) \psi^{t}(f)$, откуда получаем краевое условие для $\psi^{t}$ :

$$
\left(A_{-}-e^{i K} A_{+}-i\left(e^{i K}-1\right)(i K)^{-1} R\right) \psi^{t}=0 .
$$

Для вывода предельного уравнения учтем, что

$$
\left(\frac{\partial}{\partial x}-\frac{\partial}{\partial t}\right) I_{(-t, 0)}(x)=0 \quad \text { при } \quad x \neq 0,
$$

откуда следует, что такое же соотношение выполняется и для аргумента когерентного вектора:

$$
\left(\frac{\partial}{\partial x}-\frac{\partial}{\partial t}\right) f^{t}(x)=0
$$

Опуская промежуточные вычисления, получаем

$$
\frac{d}{d t} \psi^{t}=\left[\nabla+i\left(R^{*}\left(e^{i K}-1\right)(i K)^{-1}\right)_{1} f(t)-i\left(H_{0}\right)_{5}-\left(R^{*}(\exp \{i K\}-i K-1)(i K)^{-2} R\right)_{3}\right] \psi^{t} .
$$

Устремляя $t$ к нулю и учитывая краевое условие, имеем

$$
\begin{aligned}
& -\left.i\left(\frac{d}{d t} U(t) \psi(f)\right)\right|_{t=0} \\
& \quad=\left[-i \nabla+R^{*}\left(e^{i K}-1\right)(i K)^{-1} A_{+}+H_{0}+i R^{*}(\exp \{i K\}-i K-1)(i K)^{-2} R\right] \psi(f) .
\end{aligned}
$$

Таким образом, справедливо следующее утверждение.

Теорема 2. Сильным резольвентным пределом семейства операторов (2) при $g_{\alpha} \rightarrow \delta$ является оператор

$$
H=-i \nabla+H_{0}+R^{*}\left(e^{i K}-1\right)(i K)^{-1} A_{+}+i R^{*}(\exp \{i K\}-i K-1)(i K)^{-2} R,
$$

имеющий в качестве области определения подмножество векторов из $\mathscr{L}_{\varepsilon}(\mathscr{H})$, удовлетворяющих краевому условию (8). 
Исходя из явного вида предельного разрешающего оператора можно вывести стохастическое дифференциальное уравнение, которому оно подчиняется. Рассмотрим предел

$$
\lim _{t \rightarrow+0}(\psi(w),(U(t+d t)-U(t)) \psi(v))_{\Gamma^{S}}=(\psi(w), M(d t) \psi(v))_{\Gamma^{S}},
$$

где стохастический дифференциал $M(t)$ не должен зависеть от выбора конкретных когерентных векторов $\psi(w)$ и $\psi(v)$. Опуская промежуточные вычисления, находим выражения для стохастического дифференциала $M(d t)$ в форме Хадсона-Партасарати (см. $[4]-[6]):$

$$
\begin{gathered}
M(d t)=\left(e^{i K}-1\right) d \Lambda_{t}+\left(e^{i K}-1\right)(i K)^{-1} i R d A_{t}^{\dagger}+i R^{*}\left(e^{i K}-1\right)(i K)^{-1} d A_{t} \\
+\left(H_{0}+R^{*}\left(e^{i K}-i K-1\right)(i K)^{-2} R\right) d t
\end{gathered}
$$

Данный результат согласуется с результатами теоремы, связывающей коэффициенты исходного и предельного уравнений [7], [8].

Для формулировки основного результата введем вспомогательное понятие упорядоченного операторозначного когерентного вектора $\psi_{\mathfrak{N}}(J)$. По определению, это вектор фоковского пространства, элементами которого являются операторы некоторого гильбертова пространства $\mathscr{H}$, имеющий вид

$$
\psi_{\mathscr{N}}(H)=\overleftarrow{\exp }\left\{\int d x a^{\dagger}(x) J(x)\right\}|0\rangle, \quad J \in L_{2}(\mathscr{B}(\mathscr{H}), \mathbb{R}) .
$$

Упорядочение в хронологической экспоненте ведется по аргументам $x \in \mathbb{R}$ оператора $J(x)$. Таким образом, при действии компоненты

$$
\phi_{n}\left(x_{1} \ldots x_{n}\right)=\frac{1}{\sqrt{n !}} F\left(x_{1}\right) F\left(x_{2}\right) \cdots F\left(x_{n}\right)
$$

упорядоченного когерентного вектора $\psi(J)_{\mathscr{N}}$ на элемент гильбертова пространства $\mathscr{H}$ вначале действует оператор с наименьшим аргументом $x_{m}=\min \left(x_{1}, \ldots, x_{n}\right)$, затем оператор с аргументом $\min \left(\left\{x_{1}, \ldots, x_{n}\right\} \backslash\left\{x_{m}\right\}\right)$ и т.д. Каждая компонента такого вектора симметрична по своим аргументам: $\phi_{n}\left(\ldots x_{i} \ldots x_{j} \ldots\right)=\phi_{n}\left(\ldots x_{j} \ldots x_{i} \ldots\right)$ при $\forall n$, $1 \leqslant i<j \leqslant n$.

Получим в явном виде выражение для действия оператора $U(t)$ на упорядоченный когерентный вектор $\psi_{\mathscr{N}}(h)$ с аргументом

$$
h(x)=h_{0}(x)+h_{1}(x) I_{(-T, 0)}(x),
$$

где $h_{0}(x)$ - числовая функция, $h_{1}(x)$ - ограниченная при любом $x$ операторозначная функция со значениями в $\mathscr{B}(\mathscr{H}), T>0$. Такой вид начального состояния выбран с целью возможности проверки группового свойства разрешающего оператора. Пусть

$$
\begin{gathered}
\widehat{R}=\left(1-e^{-i K}\right)(i K)^{-1} R, \quad \widehat{R}_{2}=R^{*}(\exp \{i K\}-i K-1)(i K)^{-2} R, \\
W^{t}(x)=L^{t}(t) h(x+t) L_{-}^{t}(t) I_{\mathbb{R} \backslash(-t, 0)}(x)+V(x, t), \\
V(x, t)=L^{t}(-x) e^{i K}(h(x+t)+i \widehat{R}) L_{-}^{t}(-x) I_{(-t, 0)}(x) \\
L^{t}(z)=\overleftarrow{\exp }\left\{i \int_{t-z}^{t} d \tau\left(H_{0}+i \widehat{R}_{2}+h(\tau) \widehat{R}^{*}\right)\right\}, \quad L_{-}^{t}(z)=\left(L^{t}(z)\right)^{-1}
\end{gathered}
$$

Справедливо следующее утверждение.

Теорема 3. Предельный разрешающий оператор $U(t)$, являющийся пределом решений уравнения Шрёдингера с гамильтонианом (2), действует на когерентный вектор вида $\psi_{\mathcal{N}}(h)$, где $h$ - операторозначная функция вида (9), следующим образом:

$$
U(t) \psi(h)=\psi_{\mathscr{N}}\left(W^{t}\right) L^{t}(t)
$$


Семейство операторов $U(t)$ из (10) является унитарным коциклом: $U^{\dagger}(t)=U^{-1}(t)$, $U(s) U(t)=U(t+s)$, что проверяется непосредственными вычислениями. Вблизи нуля оператор $U(t)$ совпадает с приближенным оператором $U^{l}(t)$ :

$$
\left\|\left(U(t)-U^{l}(t)\right) \psi\right\|_{\mathfrak{H}}=O\left(t^{2}\right),
$$

для любых $\psi \in E_{\varepsilon}^{S},\|\psi\|=1$. Зная явный вид предельного разрешающего оператора для уравнения (1), найдем выражение для частичного следа произвольного оператора $B \in \mathscr{H}$ по состоянию окружения. Для произвольного когерентного вектора $\psi(h)$ из $E_{\varepsilon}^{S}$ рассмотрим частичное среднее в фоковском пространстве, являющееся элементом из $\mathscr{B}(\mathscr{H})$ :

$$
P_{t}(B)=\left(U_{t} \psi(h), B U_{t} \psi(h)\right)_{\Gamma} .
$$

Теорема 4. Функиия $P_{t}(B)$ удовлетворяет уравнению Линдблада

$$
\frac{d}{d t} P_{t}(B)=P_{t}\left(\mathscr{L}_{t}(B)\right)
$$

генератор которого имеет вид $\mathscr{L}_{t}(B)=\widehat{R}_{t}^{*} B \widehat{R}_{t}-W_{t}^{*} B-B W_{t}$, где

$$
\widehat{R}_{t}=\left(e^{i K}-1\right)(i K)^{-1} R+e^{i K} h(t), \quad W_{t}=i\left(H_{0}+(\sin K-K) K^{-2}\right)-\frac{1}{2} \widehat{R}_{t}^{*} \widehat{R}_{t} .
$$

\section{СПИСОК ЦИТИРОВАННОЙ ЛИТЕРАТУРЫ}

[1] А. М. Чеботарев, Г.В. Рыжаков, Матем. заметки, 74:5 (2003), 762-781. [2] A. M. Chebotarev, Lectures on Quantum Probability, Aportaciones Matemáticas: Textos, 14, Sociedad Matemática Mexicana, Mexico, 2000. [3] А.П. Робертсон, В. Робертсон, Топологические векторные пространства, Мир, М., 1967. [4] R. L. Hudson, K. R. Parthasarathy, Comm. Math. Phys., 93:3 (1984), 301-323. [5] K. R. Parthasarathy, An Introduction to Quantum Stochastic Calculus, Monographs in Mathematics, 85, Birkhäuser, Basel, 1992. [6] P.-A. Meyer, Quantum Probability for Probabilists, Lecture Notes in Math., 1538, Springer-Verlag, Berlin, 1993. [7] A.S. Holevo, Quantum Probability and Related Topics, 7, World Sci. Publ., Singapore, 1992, 175-202. [8] А. С. Холево, Статистическая структура квантовой теории, Институт компьютерных исследований, М.-Ижевск, 2003.

\section{Г. В. Рыжаков}

им. М. В. Ломоносова

E-mail: gryzhakov@mail.ru 